\title{
Physalins B, F and G, seco-steroids purified from Physalis angulata L., inhibit lymphocyte function and allogeneic transplant rejection
}

\author{
M.B.P. Soares ${ }^{\text {a }}$, D. Brustolim ${ }^{\text {a }}$, L.A. Santos ${ }^{\text {a }}$, M.C. Bellintani ${ }^{\text {a }}$, F.P. Paiva ${ }^{\text {a }}$, \\ Y.M. Ribeiro ${ }^{b}$, T.C.B. Tomassini ${ }^{b}$, R. Ribeiro dos Santos ${ }^{a, *}$ \\ a Centro de Pesquisas Gonçalo Moniz, FIOCRUZ, BA, Brazil \\ ${ }^{\mathrm{b}}$ FarManguinhos, FIOCRUZ, RJ, Brazil
}

Received 25 April 2005; received in revised form 20 June 2005; accepted 12 September 2005

\begin{abstract}
Physalis angulata is a solanaceae widely used in folk medicine in various tropical countries in the world. We have previously described that seco-steroids (physalins) purified from $P$. angulata are potent inhibitors of macrophage activation, blocking the production of pro-inflammatory cytokines and LPS-induced lethality. Herein we investigated the immunomodulatory activities of these substances in lymphocyte proliferation and cytokine production and in transplantation. The addition of physalins B, F or G to concanavalin A-activated splenocyte cultures induced a concentration-dependent inhibition of proliferation. Physalin B also inhibited IL-2 production by Con A-activated spleen cells. The addition of $2 \mu \mathrm{g} / \mathrm{ml}$ physalin B to mixed lymphocyte reaction (MLR) caused a $100 \%$ inhibition of proliferation. More importantly, treatment of mice with physalin B, F or G prevented the rejection of allogeneic heterotopic heart transplant. Our results demonstrate the suppressive activity of physalins B, F and G in lymphocyte function and indicate the potential use of physalins as immunosuppressive agents for treatments of pathologies in which inhibition of immune responses is desired.
\end{abstract}

(C) 2005 Elsevier B.V. All rights reserved.

Keywords: Physalin; Immunosuppression; Lymphoproliferation; Transplantation

\section{Introduction}

Immunosuppressive substances are widely used to inhibit undesired immune responses in situations such as autoimmune diseases, allergies and organ transplantations. Although a variety of immunosuppressive

\footnotetext{
* Corresponding author. Centro de Pesquisas Gonçalo Moniz, FIOCRUZ, Rua Waldemar Falcão, 121 - Candeal - Salvador, BA, Brazil, CEP: 40296-750. Tel.: +55 $7133564320 \times 260 / 272$; fax: +55713356 2155.

E-mail address: ricardoribeiro@cpqgm.fiocruz.br (R. Ribeiro dos Santos).
}

drugs is currently available, their prolonged use is usually accompanied by undesired effects. Among the most employed immunosuppressors are the glucocorticoids, administered alone or in combination with other agents [1]. Glucocorticoids have a broad spectrum of actions, causing down-regulation of pro-inflammatory cytokines such as TNF- $\alpha$, IL-1, IL-6 and IL-12, inhibition of lymphocyte proliferation and decreased monocyte and neutrophyl chemotaxis [2]. Despite the broad use of glucocorticoids and its important contribution to the establishment of organ transplantation protocols, life-long use of these substances causes severe adverse effects in many patients, such as osteopenia, poor 
wound healing, hyperglycemia, hypertension and cataracts [2]. Thus, the finding of substances with similar immunosuppressive activities with no or lower toxicity is of great interest.

Natural products have been a source of compounds with pharmacological activities. Physalis angulata L. is a plant widely distributed throughout the tropical and subtropical regions of the world. The broad use of this plant in popular medicine includes wound healing, hepatitis, malaria, sleeping sickness, rheumatism, earache, fever, among others [3-6]. A series of secosteroids known as physalins, were isolated from Physalis $\mathrm{sp}$. and characterized [7-10]. Immunomodulatory activity of fractions prepared from $P$. angulata extracts has been previously described [11].

In a previous study we demonstrated that physalins purified from $P$. angulata have suppressive activity on macrophage cultures stimulated with lipopolysaccharide and interferon- $\gamma$ [12]. Administration of physalins $B, F$ or $G$ prevented mortality induced by a lethal injection of LPS in mice [12]. In the present work we tested whether these seco-steroids purified from $P$. angulata modulate lymphocyte functions in lymphoproliferative and cytokine production assays and in allogeneic transplantation model.

\section{Materials and methods}

\subsection{Mice}

Male and female 6-8 weeks old BALB/c, CBA, C3H and $\mathrm{C} 57 \mathrm{BL} / 6$ mice were used as spleen cell donors and as recipients of allogeneic grafts. Newborn CBA mice were used as heart donor in transplantation studies. All mice were raised and maintained at the animal facilities at Gonçalo Moniz Research Center, FIOCRUZ (Salvador, Brazil), provided rodent diet and water ad libitum. All mice were sacrificed under anaesthesia and treated according to the Oswaldo Cruz Foundation guidelines for laboratory animals.

\subsection{Physalin preparations}

Physalins B, D, F and G (Fig. 1) were purified from stems of dried P. angulata plants collected in Belém do Pará, Brazil. P. angulata was identified by Dr. Lucia Carvalho from Botanical Garden of Rio de Janeiro. A voucher specimen is held under number RFA 23907/8 at Federal University of Rio de Janeiro, Brazil. Purification was carried out according to previously described methodology [12]. The purity of physalins was determined by HPLC technique and the average range was between $96 \%$ and $98 \%$ for the seco-ergostane derivatives [12]. The physalins are stable at room temperature $\left(30-33{ }^{\circ} \mathrm{C}\right)$, kept in amber flask, and are soluble in ethyl acetate and methanol. Preparations
Physalin B

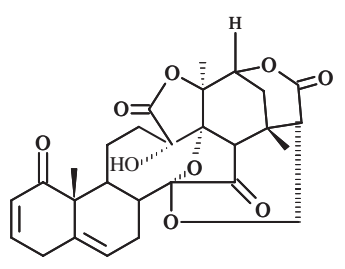

Physalin F

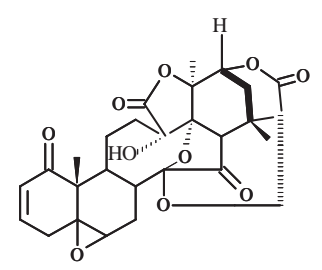

Physalin D

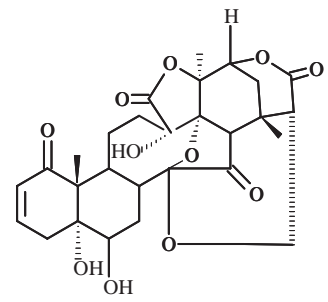

Physalin G

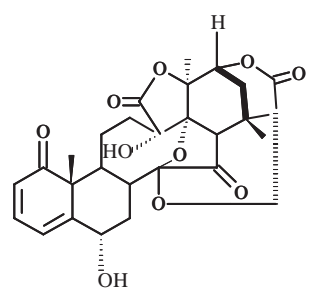

Fig. 1. Chemical structures of physalins B, D, F and G.

of pure physalins were dissolved in dimethyl sulfoxide (DMSO) and diluted in culture medium for use in the assays.

\subsection{Mitogen-induced lymphoproliferation assay}

BALB/c splenocyte suspensions were prepared in RPMI medium (Life Technologies, GIBCO-BRL, Gaithersburg, MD, USA) supplemented with $10 \%$ fetal calf serum (Hyclone, Logan, UT, USA), $2 \mathrm{mM}$ of L-glutamine, vitamins, $1 \mathrm{mM}$ of sodium pyruvate, $10 \mathrm{mM}$ of HEPES, $50 \mu \mathrm{M}$ of 2mercaptoethanol, and $50 \mu \mathrm{g} / \mathrm{ml}$ of gentamycin (Sigma, St. Louis, MO, USA). Splenocytes were cultured in 96-well plates at $4 \times 10^{5}$ cells/well in $200 \mu \mathrm{l}$, in triplicate wells, in the presence of concanavalin A (Con A), alone or in various concentrations of physalin preparations, as described in figure legends. After $48 \mathrm{~h}$, plates were pulsed with $1 \mu \mathrm{Ci}$ of $\left[\right.$ methyl $\left.-{ }^{3} \mathrm{H}\right]$ thymidine (Amersham, Little Chalfont, England) for $12 \mathrm{~h}$, and proliferation was assessed by measurement of ${ }^{3} \mathrm{H}-$ thymidine uptake in a $\beta$-plate counter (Packard, Meriden, CT, USA). In some experiments, dexamethasone, a synthetic glucocorticoid, and RU486, a glucocorticoid receptor antagonist (Sigma), were added to the cultures to investigate the mechanism of physalins' action. To rule out the possibility of a toxic effect of physalin preparations, cell viability was monitored by counting the number of trypan blue-stained cells, after $48 \mathrm{~h}$ of culture with Con A alone or in combination with physalin B, by optical microscopy.

\subsection{Mixed lymphocyte reaction}

BALB/c $\left(H-2^{\mathrm{d}}\right)$ mice were weekly immunized with ficollpurified C57BL/6 $\left(H-2^{\mathrm{b}}\right)$ splenocytes i.p. $\left(10^{7}\right.$ cells/mouse), respectively. After 3 weeks of immunization, mice were sacrificed for spleen cell preparation in RPMI medium supple- 
mented as described above. Spleen cells were cultured in 96 well plates at $5 \times 10^{5}$ cells/well in the absence or in the presence of irradiated C57BL/6 splenocytes at $10^{6}$ cells/well (dose of 3000 rads in a ${ }^{137} \mathrm{Cs}$ source irradiator purchased from CisBio International, France) and different physalin preparations, in triplicates. After $72 \mathrm{~h}$ of culture, plates were pulsed with ${ }^{3} \mathrm{H}$-thymidine for $12 \mathrm{~h}$ for proliferation assessment.

\subsection{Cytokine determination}

For cytokine determination, spleen cells obtained from normal BALB/c mice $\left(5 \times 10^{6}\right.$ cells $\left./ \mathrm{ml}\right)$ were cultured in supplemented RPMI medium in 24 well plates and stimulated with $1 \mu \mathrm{g} / \mathrm{ml}$ of Con A. Cell-free supernatants were collected after $24 \mathrm{~h}$ of incubation and stored at $-20{ }^{\circ} \mathrm{C}$ for cytokine analysis. Levels of IL-2 in culture supernatants were determined by sandwich ELISA, using antibody pairs and recombinant IL-2 from PharMingen, following manufacturer's instructions. Reaction was developed using the 3,3',5,5' -tetramethylbenzidine (TMB) peroxidase substrate (Kinkergaard and Perry Laboratories, Gaithersburg, MD, USA) and read at $450 \mathrm{~nm}$.

\subsection{Heterotopic heart transplant}

We have used the technique of heart transplantation described previously [13]. Newborn CBA mice were briefly anesthetized by hypothermia for immediate excision of the heart followed by implantation into the dorsal base of the pinna of the ear of male BALB/c mice. Mice received two grafts, one in each ear. The implant was subcutaneous, and the surgical incision was gently closed by pressure with the aid of forceps. Mice were orally treated with vehicle, physalin B, F or $\mathrm{G}(1 \mathrm{mg} / \mathrm{mouse} /$ day $)$ during 30 days starting from the transplantation day. The dose of physalin used in this work does not cause any acute toxic effects, which are observed only with doses equal or higher than $8 \mathrm{mg} / \mathrm{mouse} /$ day (our unpublished results). Grafts were observed using a stereoscopic microscope (Olympus, Japan) every 5 days. Rejection was defined as the disappearance of the grafted tissue.

\subsection{Statistical analyses}

In vitro experiments were analyzed using one-way ANOVA followed by Newman-Keuls multiple comparison test, using Graph Pad Prism 3.0 software. Graft survival curves
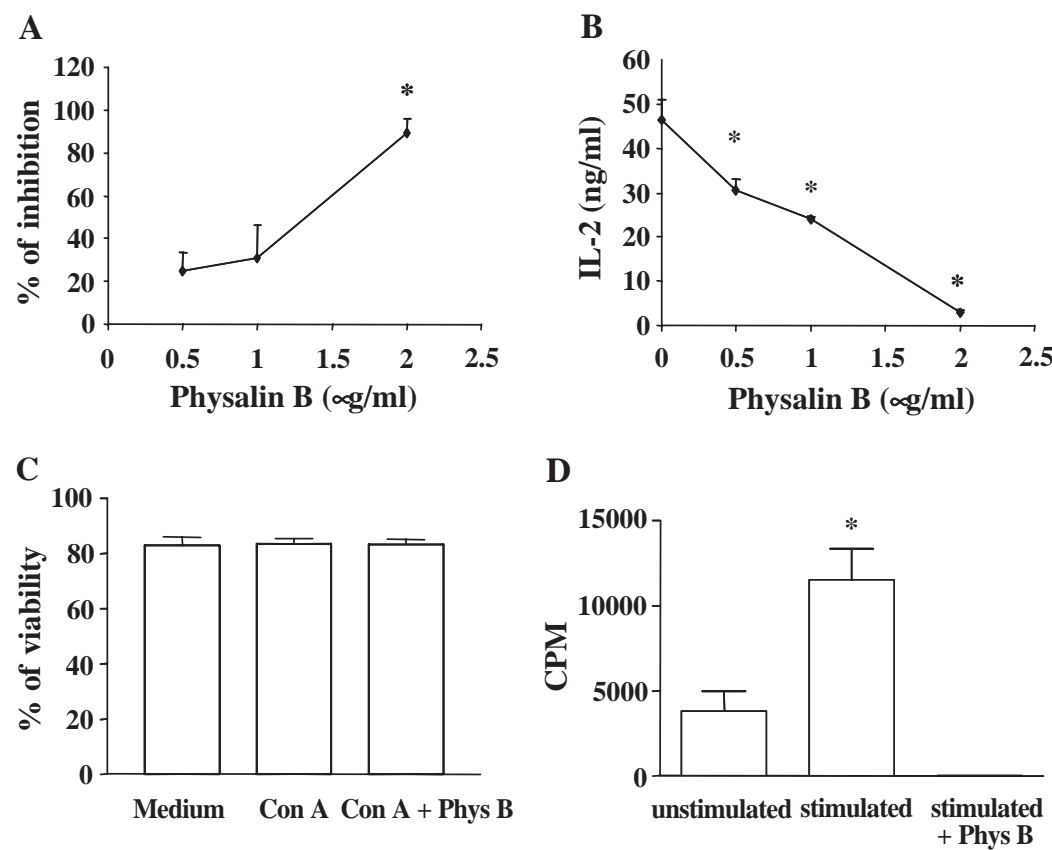

Fig. 2. Inhibition of Con A-induced lymphocyte activation by physalin B. BALB/c spleen cell were stimulated in vitro with $1 \mu \mathrm{g} / \mathrm{ml}$ of Con $\mathrm{A}$ in the absence or in the presence of different concentrations of physalin B. The proliferative response (A) and IL-2 production (B) were determined after 48 and $24 \mathrm{~h}$ of culture, respectively. (C) Spleen cells were incubated without (medium) or with Con A and physalin B (Phys B; $2 \mu \mathrm{g} / \mathrm{ml}$ ). Cell viability was determined $48 \mathrm{~h}$ after incubation by trypan blue exclusion. Values represent the means \pm S.E.M. of the percentage of inhibition in relation to untreated, Con A-stimulated cultures, obtained in 5 independent experiments (A) or the means \pm S.D. of 3 determinations (B and C). (D) Splenocytes from BALB/c mice sensitized with C57BL/6 cells were cultured in the absence (unstimulated) or presence (stimulated) of irradiated C57BL/6 spleen cells, with or without physalin B $(2 \mu \mathrm{g} / \mathrm{ml})$. Proliferation was assessed $72 \mathrm{~h}$ later by ${ }^{3} \mathrm{H}$-thymidine incorporation. Values represent the means \pm S.E.M. of 5 mice. ${ }^{*} P<0.05$ compared to untreated controls. 


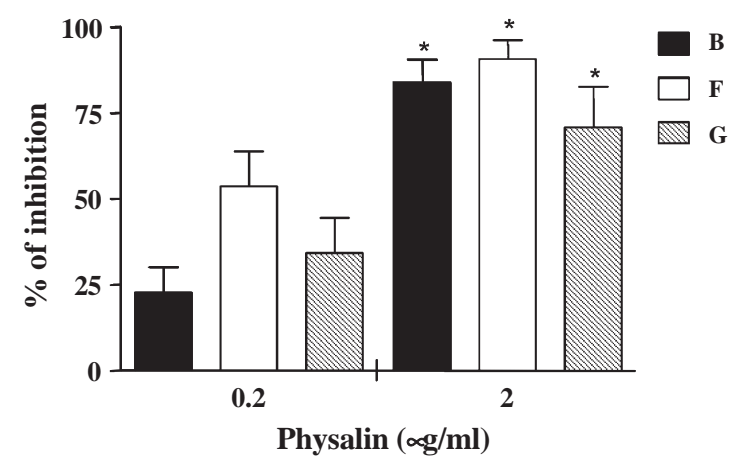

Fig. 3. Comparison of Con A-induced lymphoproliferation inhibition by different physalins. Con A-stimulated splenocyte cultures were incubated with physalins $B, F$ and $G$ at 2 and $0.2 \mu \mathrm{g} / \mathrm{ml}$. The percentage of inhibition was calculated in relation to untreated, Con A-stimulated cultures. Values represent the means \pm S.D. of 7 independent experiments. ${ }^{*} P<0.05$ compared to $0.2 \mu \mathrm{g} / \mathrm{ml}$ of physalins.

were analyzed according to the Kaplan-Meier method using SPSS software version 9.0. Differences were considered significant when $P<0.05$.

\section{Results}

\subsection{Physalins inhibit concanavalin A-induced proliferation and IL-2 secretion by mouse splenocytes}

Addition of physalin B in cultures of BALB/c splenocytes stimulated with Con A caused a concentration-dependent inhibition of proliferation and of IL-2 production (Fig. 2A and B). Physalin B caused an inhibition of $90-100 \%$ at a concentration of $2 \mu \mathrm{g} / \mathrm{ml}$. Cell viability in physalin B-treated wells was similar to that of untreated cultures, indicating that in vitro inhibition of lymphocyte function was not due to cytotoxic effects of physalin preparation (Fig. $2 \mathrm{C} ; P>0.05$ ). Addition of physalins $\mathrm{F}$ and $\mathrm{G}$ also caused the inhibition of
Con A-induced proliferation of splenocytes in a dose-dependent manner (Fig. 3). Another seco-steroid tested, physalin D, had no effects on the inhibition of Con A-stimulated lymphoproliferation (data not shown). Addition of $2 \mu \mathrm{g} / \mathrm{ml}$ of physalin B also caused a $90-100 \%$ inhibition of proliferation in MLR cultures of spleen cells of BALB/c mice stimulated with C57BL/6 cells (Fig. 2D).

\subsection{Inhibition of Con A-induced lymphoproliferation by physalin $B$ is not reverted by the addition of RU486}

To investigate if physalin B acts through the activation of the glucocorticoid receptor, we tested whether the addition of RU486, a glucocorticoid receptor antagonist, affected physalin B-induced inhibition of lymphoproliferation (Fig. 4). Addition of RU486 to physalin B-treated, Con A-stimulated spleen cell cultures did not decrease de inhibition of proliferation; inhibition was even higher in cultures treated with physalin B and RU486 (Fig. 4). In contrast, the inhibition of spleen cell proliferation upon Con A activation by dexamethasone, a synthetic glucocorticoid with immunomodulatory activity, was blocked by addition of RU486 (Fig. 4).

\subsection{Rejection of allogeneic heterotopic heart transplant is delayed by treatment with physalins}

$\mathrm{BALB} / \mathrm{c}$ mice were transplanted with hearts of newborn CBA mice into the ear pinna. Groups of transplanted BALB/c mice were treated with $1 \mathrm{mg} /$ day/mouse of physalins $\mathrm{B}, \mathrm{F}$ or $\mathrm{G}$ or vehicle during 30 days (Fig. 5). 15 days after transplantation, only $2 / 6$ vehicle-treated mice had viable grafts, whereas all mice $(6 / 6)$ of the groups treated with the different physalins had viable grafts at this time-point. Vehicle-treated mice had no viable grafts after 25 days of transplantation, a time-point when $50-66.6 \%$ of physalin-treated mice had viable grafts. Graft survival curves of animals treated with any of the three physalins were significantly different from that of

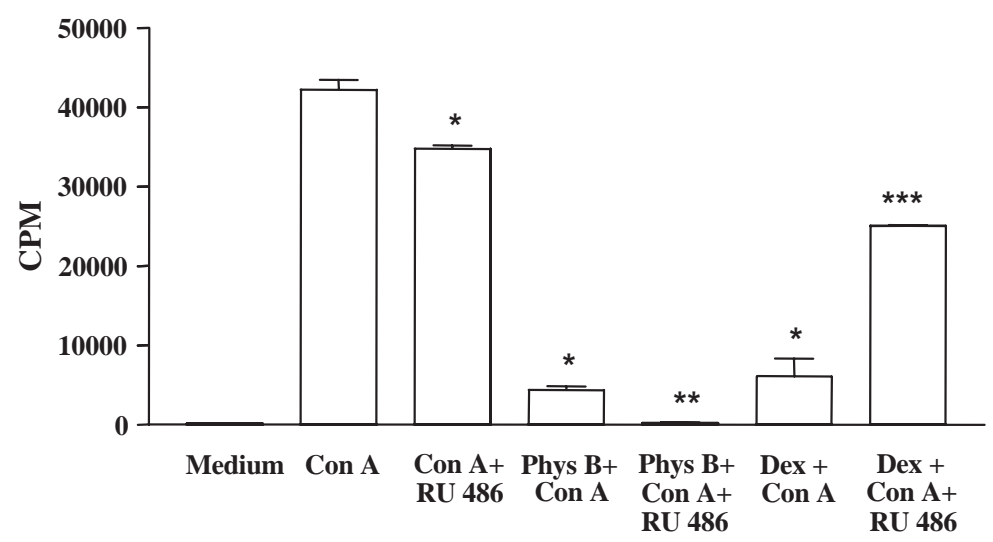

Fig. 4. Effects of RU486 on physalin B activity on Con A-induced lymphoproliferation. BALB/c splenocytes were stimulated with Con A alone or in the presence of dexamethasone (Dex; $1 \mu \mathrm{M}$ ), Physalin B (Phys B; $2 \mu \mathrm{g} / \mathrm{ml}$ ) and RU486 $\left(10^{-5} \mathrm{M}\right.$ ). Proliferation was assessed $48 \mathrm{~h}$ later by ${ }^{3} \mathrm{H}-$ thymidine incorporation. Values represent the means \pm S.D. of 3 determinations. ${ }^{*} P<0.001$ compared to Con A; $* * P<0.05$ compared to Phys $\mathrm{B}+\mathrm{Con} \mathrm{A} ; * * * P<0.001$ compared to $\mathrm{Dex}+\mathrm{Con} \mathrm{A}$. 


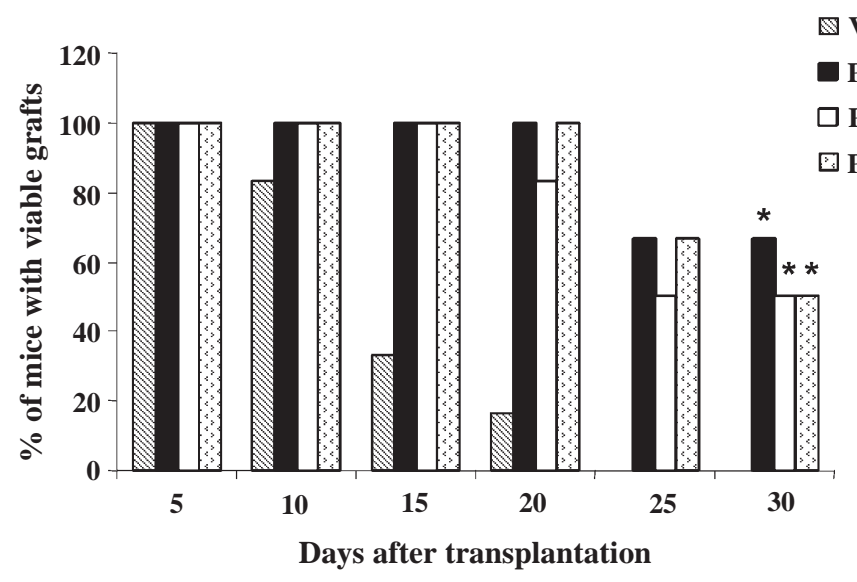

Fig. 5. Treatment with physalins prevents allogeneic transplant rejection. Groups of $6 \mathrm{BALB} / \mathrm{c}$ mice receiving heterotopic CBA newborn hearts transplanted into each ear were treated daily with physalin B, F or G (1 mg/mouse/day) or with vehicle starting from the transplantation day. Values represent the percentage of mice with at least one viable graft of 6 mice.
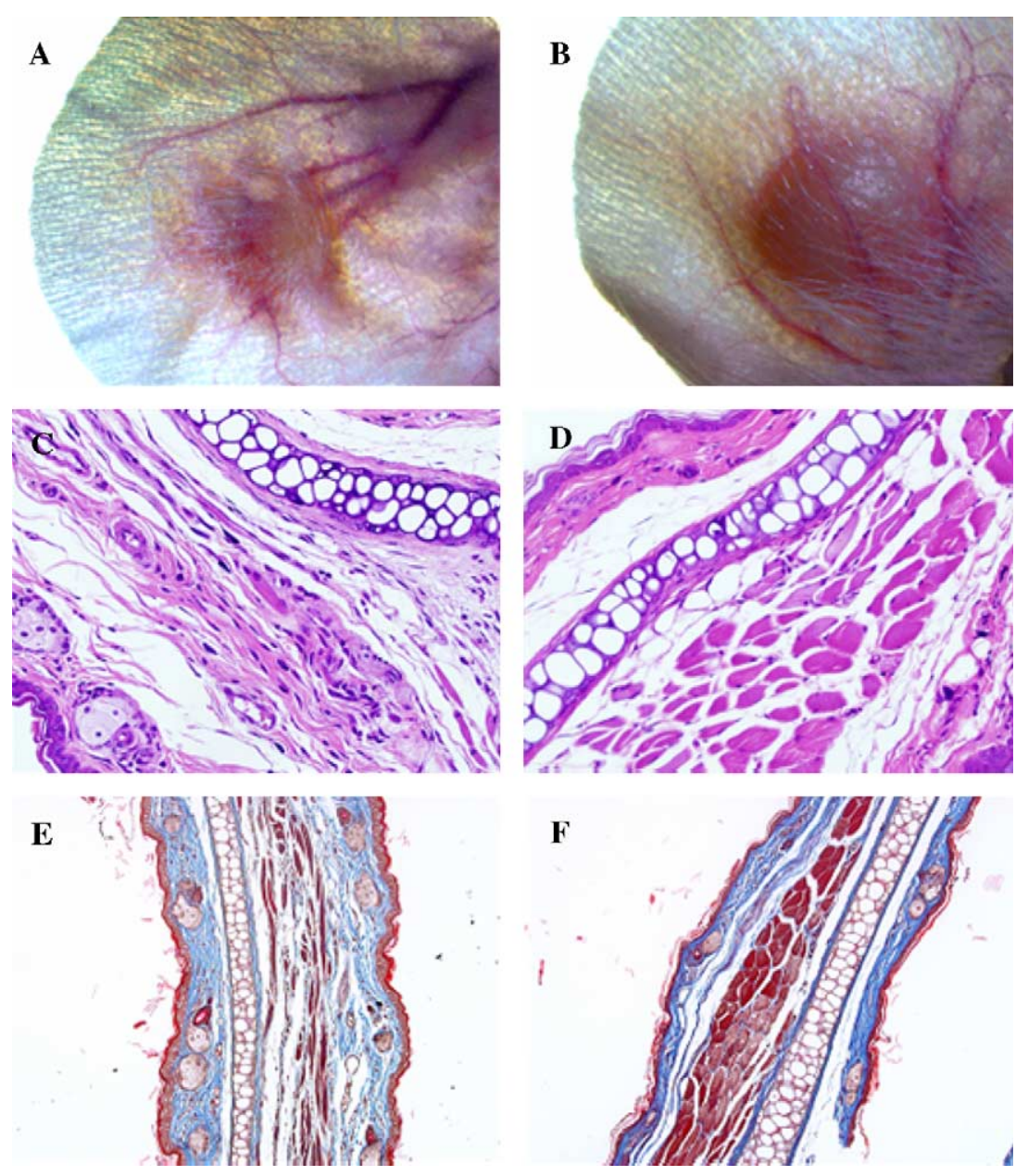

Fig. 6. Effects of physalin treatment of allogeneic heart transplant. Heart transplants of mice treated with vehicle (A) or with physalin G (B) 30 days after transplantation. Ear sections of vehicle-treated (C and E) and physalin B-treated (D and F) mice. (C and D) H\&E stain, 20× objective. (E and F) Masson's trychrome stain, $20 \times$ objective. 
control mice $(P<0.05$, Kaplan-Meier method). 30 days after transplantation, rejected grafts from vehicle-treated mice were absorbed (Fig. 6A), and inflammation and fibrotic areas associated with necrotic myofibers were observed in the transplantation site (Fig. 6C and E). In contrast, viable grafts from physalin-treated mice had vascularization (Fig. 6B), discrete inflammation and intact myofibers (Fig. 6D and F).

\section{Discussion}

In the present report we demonstrated that physalins $B, F$ and $G$ have potent suppressive activities in vitro on splenocyte cultures and in vivo on allogeneic transplant. These observations confirm and extend the immunomodulatory action of these seco-steroids purified from $P$. angulata recently reported [12]. A fourth molecule, physalin D, was also tested, but had no effects on Con A-stimulated proliferation. In our previous report, this physalin was also inactive on activated macrophage cultures [12].

The suppression by physalins was evidenced in vitro by the inhibition of lymphocyte proliferation and IL-2 production. Physalins inhibited both mitogen-stimulated as well as allospecific lymphocyte proliferation, indicating that inhibition was not a non-specific phenomenon due to interference on the interaction of Con A with surface molecules on spleen cells. The fact that physalins $\mathrm{B}$ and $\mathrm{F}$ have inhibitory effects on the growth of several leukemic cell lines, including acute $\mathrm{T}$ and $\mathrm{B}$ lymphoid leukemia cell lines [14], suggests that these molecules interfere directly with lymphocyte proliferation.

Acute allogeneic transplant rejection is an inflammatory response triggered by allospecific lymphocytes. Therefore, the effects of physalins on transplant rejection could be explained by a direct effect of these substances on lymphocytes. However, as shown in a previous study [12], these physalins also inhibit the production of pro-inflammatory cytokines and macrophage activation. Thus, the inhibition of allogeneic graft rejection by physalin treatment, similar to the action of glucocorticoids, could also be the result of the inhibition of responses by non-lymphocyte inflammatory cells such as macrophages, in addition to the inhibition of lymphocytes. Both activities of physalins could contribute to a decreased inflammation and fibrosis deposition observed on the transplantation site of viable grafts in physalin-treated mice, compared to vehicle-treated mice.

The probable pathway of physalins action is the activation of endogenous steroid receptors in the cells which will act as gene regulators. Different from dexamethasone, a gold standard glucocorticoid, the effects of physalins on Con A-stimulated lymphocytes were not blocked by the glucocorticoid receptor antagonist RU486. The failure of RU486 to inhibit physalins actions was observed before on activated macrophage cultures [12]. Whether physalins act by activating glucocorticoid receptor through a different mechanism or by activating a different receptor is under investigation.

$P$. angulata extracts are widely employed in many tropical countries throughout the world as traditional remedies for various diseases, such as sleeping sickness, gonorrhea and malaria [3-6]. The activities of physalins described in this and in a previous work [12] suggest that some of the effects observed in folk medicine with the use of $P$. angulata extracts in the treatment of several illnesses are in part due to the action of these seco-steroids with immuno-inflammatory action present in this plant species.

In conclusion, our study indicates that physalin administration may be useful in the treatment of various immune-mediated pathologies, such as autoimmune and allergic diseases and on the prevention of transplant rejection. Its use will be especially relevant if these phytosteroids have lower toxic effects when compared to conventional steroidal immunosuppressive drugs such as glucocorticoids, an issue that we are currently investigating.

\section{Acknowledgements}

This work was supported by grants from (CNPq), Instituto do Milênio do Semi-Árido (IMSEAR-MCT) and FIOCRUZ (PDTIS). The authors wish to thank Drs. Augusto C. A. Mota and Washington L. C. Santos who kindly helped with the statistical analyses.

\section{References}

[1] Krensky AM, Weiss A, Crabtree G, Davis MM, Parham P. Immunomodulators: immunosuppressive agents, tolerogens, and immunostimulants. In: Hardman JG, Limbird LE, Goodman Gilman A, editors. The pharmacological basis of therapeutics, 10th ed. New York: McGraw-Hill; 2001. p. 1463-83.

[2] Schimmer BP, Parker KL. Adrenocorticotropic hormone; adrenocortical steroids and their synthetic analogs; inhibitors of the synthesis and actions of adrenocortical hormones. In: Hardman JG, Limbird LE, Goodman Gilman A, editors. The pharmacological basis of therapeutics, 10th ed. New York: McGraw-Hill; 2001. p. 1649-77.

[3] Caceres A, Menendez H, Mendez E, Cohobon E, Samayoa BE, Jauregui E, et al. Antigonorrhoeal activity of plants used in Guatemala for the treatment of sexually transmitted diseases. J Ethnopharmacol 1995;48(2):85-8.

[4] Ankrah NA, Nyarko AK, Addo PG, Ofosuhene M, Dzokoto C, Marley E, et al. Evaluation of efficacy and safety of a herbal medicine used for the treatment of malaria. Phytother Res 2003;17(6):697-701. 
[5] Freiburghaus F, Kaminsky R, Nkunya MH, Brun R. Evaluation of African medicinal plants for their in vitro trypanocidal activity. J Ethnopharmacol 1996 (Dec);55(1):1-11.

[6] Choi EM, Hwang JK. Investigations of anti-inflammatory and antinociceptive activities of Piper cubeba, Physalis angulata and Rosa hybrida. J Ethnopharmacol 2003;89(1):171-5.

[7] Matsuura T, Kawai M, Nakashima R, Butsugan Y. Structures of physalin A and physalin B, 13,14-Seco-16,24-cyclo-steroids from Physalis alkekengi var. Francheti. J Chem Soc 1970;5:664-70.

[8] Mulchandani NB, Iyer SS, Badheka LP, Physalin D. A new 1314-Seco-16,24-cyclo steroid from Physalis minima. Planta Med 1979;37:268-73.

[9] Row LR, Sarma NS, Reddy KS, Matsuura T, Nakashima R. The structures of physalins $\mathrm{F}$ and $\mathrm{J}$ from Physalis angulata $\mathrm{L}$. and Physalis lancifolia. Phytochemistry 1978;17:1647-50.

[10] Row LR, Reddy KS, Sarma NS, Matsuura T, Nakashima R. New physalins from Physalis angulata L. and Physalis lancifo- lia structures and reactions of physalins D, I, G and K. Phytochemistry 1980;17:1175-81.

[11] Lin YS, Chiang HC, Kan WS, Hone E, Shih SJ, et al. Immunomodulatory activity of various fractions derived from Physalis angulata L. extract. Am J Chin Med 1992;20:233-43.

[12] Soares MB, Bellintani MC, Ribeiro IM, Tomassini TC, Ribeiro dos Santos R. Inhibition of macrophage activation and lipopolysaccaride-induced death by seco-steroids purified from Physalis angulata L. Eur J Pharmacol 2003;459(1):107-12.

[13] dos Santos RR, Rossi MA, Laus JL, Silva JS, Savino W, Mengel J. Anti-CD4 abrogates rejection and reestablishes long-term tolerance to syngeneic newborn hearts grafted in mice chronically infected with Trypanosoma cruzi. J Exp Med 1992;175(1): 29-39.

[14] Chiang HC, Jaw SM, Chen PM. Inhibitory effects of physalin B and physalin $\mathrm{F}$ on various human leukemia cells in vitro. Anticancer Res 1992;12(4):1155-62. 